\title{
Cardiometabolic comorbidities in obstructive sleep apnea patients are related to disease severity, nocturnal hypoxemia, and decreased sleep quality
}

Stephanie André1,2,3, Fabio Andreozzi ${ }^{1,2}$, Chloé Van Overstraeten ${ }^{1,2}$, Sidali Ben Youssef ${ }^{1,2}$, lonela Bold ${ }^{1,2}$, Sarah Carlier ${ }^{1,2,3}$, Alexia Gruwez ${ }^{1,2,3}$, Anne-Violette Bruyneel ${ }^{4}$ and Marie Bruyneel ${ }^{1,2,3^{*}}$ (D)

\begin{abstract}
Background: Obstructive sleep apnea syndrome (OSA) is currently recognized as an independent risk factor for hypertension, arrhythmia, coronary heart disease, stroke, and metabolic disorders (e.g. diabetes, dyslipidemia). In clinical practice, apnea-hypopnea index (AHI) is the marker used to classify disease severity and guide treatment. However, AHI alone does not sufficiently identify OSA patients at risk for cardiometabolic comorbidities. With this in mind, the aim of this retrospective study was to determine whether some polysomnographic parameters (e.g. apnea-hypopnea duration, sleep structure, nocturnal hypoxemia) are specifically associated with cardiometabolic comorbidities in OSA.

Methods: In this retrospective study, 1717 patients suffering from moderate/severe OSA were included between 2013 and 2017. Data on demographics, comorbidities, and polysomnographic characteristics were collected and analyzed to identify factors associated with cardiometabolic complications.

Results: The medical files of 1717 patients (68\% male) were reviewed. The mean AHI was $43.1+/-27.7$ with 57.3\% of patients suffering from severe OSA, and 52\% from at least one cardiovascular comorbidity (CVCo). Diabetes affected $22 \%$ of the patients and $27 \%$ exhibited dyslipidemia. Patients affected by CVCos were older, and more often women and non-smokers. These patients also had worse sleep quality, and a more marked intermittent/ global nocturnal hypoxemia. With regard to diabetes, diabetics were older, more often non-smoker, non-drinker women, and were more obese. These patients also exhibited more severe OSA, especially in non-REM (NREM) sleep, worse sleep quality, and a more marked intermittent/global nocturnal hypoxemia. Dyslipidemia was more frequent in the absence of alcohol consumption, and was associated with OSA severity, decreased sleep quality, and longer AH in REM sleep.

Conclusions: This study identifies demographic and polysomnographic factors associated with cardiometabolic comorbidities. Patients (especially women) suffering from more severe OSA, longer sleep apneas and hypopneas, worse sleep quality, and marked intermittent/global nocturnal hypoxemia are more likely to develop cardiometabolic comorbidities. This should stimulate clinicians to obtain adequate treatment in this population.
\end{abstract}

Keywords: Diabetes, Cardiovascular comorbidities, Dyslipidemia, Obstructive sleep apnea, Nocturnal hypoxemia, Sleep quality, Polysomnography

\footnotetext{
* Correspondence: Marie_Bruyneel@stpierre-bru.be

${ }^{1}$ Department of Pulmonary Medicine, Saint-Pierre University Hospital, Rue

Haute 322, 1000 Brussels, Belgium

${ }^{2}$ Université Libre de Bruxelles, Brussels, Belgium

Full list of author information is available at the end of the article
}

(c) The Author(s). 2020 Open Access This article is distributed under the terms of the Creative Commons Attribution 4.0 International License (http://creativecommons.org/licenses/by/4.0/), which permits unrestricted use, distribution, and reproduction in any medium, provided you give appropriate credit to the original author(s) and the source, provide a link to the Creative Commons license, and indicate if changes were made. The Creative Commons Public Domain Dedication waiver (http://creativecommons.org/publicdomain/zero/1.0/) applies to the data made available in this article, unless otherwise stated. 


\section{Background}

Obstructive sleep apnea (OSA) syndrome is a common medical problem. The prevalence of the disorder is increasing and is related to its main causal factors, namely obesity and aging [1]. OSA is now recognized as an independent risk factor for hypertension, arrhythmia, coronary heart disease, and stroke [2-4]. Metabolic disorders (diabetes, impaired lipid metabolism) are also associated with OSA $[5,6]$.

Despite these associations, the clinical picture of OSA is not homogeneous and can differ widely among patients. In the last decade, a variety of clinical phenotypes have been identified, highlighting clusters with different symptomologies and morbidities in patients affected by the "same" OSA [7]. Response to treatment is also impacted by these clinical phenotypes. For example, Gagnadoux et al. showed that clusters defined as "female OSA", "mildly symptomatic OSA", and "comorbid OSA" were less likely to use continuous positive airway pressure (CPAP) successfully [8].

In clinical practice, apnea-hypopnea index (AHI) is still the marker used to classify disease severity and guide treatment, but it is a limited marker that doesn't sufficiently describe the clinical picture of OSA. For example, among patients with the same AHI, some exhibit long apneas with profound oxygen desaturation, and others very short apneas without significant associated hypoxia. These differences can potentially lead to several clinical OSA phenotypes that should maybe not be managed similarly.

With this in mind, the aim of this retrospective study was to identify whether some particular polysomnographic parameters (e.g. apnea-hypopnea duration, sleep structure, nocturnal hypoxemia) are specifically associated with cardiometabolic comorbidities in OSA patients.

\section{Methods}

This study was performed in the sleep unit of the SaintPierre University Hospital in Brussels, Belgium (tertiary referral center).

\section{Study design}

The study was retrospective, based on prospectively collected data in our sleep lab. All naïve patients suffering from moderate-to-severe sleep apnea syndrome (apneahypopnea index (AHI) $\geq 15 /$ h of sleep) diagnosed by one single attended polysomnography (PSG) between 01/01/ 2013 and 12/31/2017 were included.

Data for medical history, treatments, and polysomnographic characteristics were extracted from patient medical files.

Polysomnographies were all performed with the same polysomnographic device in the sleep laboratory of $\mathrm{CHU}$ St. Pierre (Brainnet3 IP, Medatec, Belgium).

\section{Data collection}

Age, sex, body mass index (BMI), neck circumference (NC), and alcohol and tobacco use were collected.

Medical files were analyzed in order to collect data on comorbidities (e.g. arterial hypertension (HTA), diabetes, atrial fibrillation). Congestive heart failure was defined by a left ventricular ejection fraction below $35 \%$. PSG was scored according to American Academy of Sleep Medicine 2012 scoring rules [9] and reviewed in order to collect, for all patients, AHI (global, supine/ non supine, rapid eye movement (REM) sleep), mean sleep apnea/hypopnea (AH) duration (according to sleep stage), total sleep time (TST), sleep efficiency (SE), sleep stage proportions, periodic leg movements (PLM), oxygen desaturation index (ODI), total duration of oxygen desaturation $<90 \%$, arousal index (ARI), minimal oxygen $\left(\mathrm{O}_{2}\right)$ saturation, and mean nocturnal heart rhythm (HR).

Supine predominant OSA was considered when AHI supine was $\geq$ two-fold non-supine [10], and REM Predominant when AHI in REM sleep was $\geq$ two-fold more than in non-REM sleep [11].

The study protocol was approved by the Saint-Pierre University Hospital ethics committee (CE/18-01-06).

\section{Statistical analysis}

Statistical analyses include descriptive statistics. Characteristics of the study population are presented as means with standard deviations for quantitative data and as percentages for qualitative data for the entire cohort and sub-groups. We compared data according to the presence of diabetes (with/without diabetes), cardiovascular comorbidities (with/without), and dyslipidemia (with/ without).

In order to compare baseline parameters between groups, an Analysis of variance (ANOVA) with Newman Keuls post-hoc test for quantitative variables (BMI, neck circumference, alcohol, and PSG parameters) or chi-square tests for qualitative variables (sex, ethnicity, smoking, supine, and REM predominant) were applied to compare demographic and polysomnographic data.

To explore possible factors that were associated with diabetes, cardiovascular comorbidities, and dyslipidemia, logistic regression analysis or multiple regression analysis were also used. The regression was adjusted by age, BMI, and smoking status for diabetes, by age, BMI, smoking, sex, and dyslipidemia for cardiac comorbidities, and by age, sex, and BMI for dyslipidemia.

A $p$ value below 0.05 was considered as statistically significant. All analyses were performed using Statistica software (v. 6, StatsoftTM) and SPSS version 22.0 (SPSS Inc., Chicago, IL, USA). 


\section{Results}

\section{Demographics}

The study included 1717 patients, 1144 males and 573 females. The mean age for the whole population was $52.8 \pm 12.7$ years old. Females were slightly older than males (females: $54.35 \pm 12.76$ y vs. males: $52.08 \pm 12.62$ y). A total of $68 \%$ of patients were obese. Patient characteristics are summarized in Table 1.

\section{Polysomnography}

The mean apnea-hypopnea index (AHI) was $43.1 \pm 25.7$ with $57.30 \%$ of patients suffering from severe OSA (AHI tot $>30$ ).

\section{Comorbidities}

Fifty-two percent of patients suffered from at least one cardiovascular comorbidity (HTA, atrial fibrillation, ischemic cardiomyopathy, congestive heart failure (left ventricular ejection fraction $<35 \%$ ), or cerebral stroke). Metabolic comorbidities were also frequent, as diabetes affected $22 \%$ of patients and dyslipidemia 27\% (Fig. 1).

Patients received pharmacological treatment accordingly but compliance to prescribed medications remained unknown.

\section{Diabetes}

Comparing diabetics and non-diabetics, diabetics were older, more often non-smoker non-drinker women, but more obese. They also exhibited more severe OSA, especially in non-REM (NREM) sleep, worse sleep quality (reflected by low SE, TST, percentage REM sleep on TST), more marked intermittent (reflected by ODI) and global (reflected by mean oxygen saturation, oxygen desaturation,

Table 1 Demographic characteristics of the patients

\begin{tabular}{ll}
\hline & $\begin{array}{l}\text { Study Group } \\
N=1717\end{array}$ \\
\hline Age (years) Mean \pm SD & $54.14 \pm 12.76$ \\
Sex (\%) & Female: 32.46 \\
& Male: 67.54 \\
BMI (kg/m²) Mean \pm SD & $34.29 \pm 8.42$ \\
NC (cm) Mean \pm SD & $42.81 \pm 4.84$ \\
Smokers (\%) & Current smokers: 25.40 \\
& Non-smokers: 74.60 \\
& Former smokers: 4.61 \\
Alcohol (\%) & Non-drinkers: 39.79 \\
& Drinkers: 60.21 \\
& StD:24.18 \\
Unit/day Mean \pm SD & LD:4.80 \\
\hline
\end{tabular}

SD Standard deviation, BMI Body mass index, NC Neck circumference, StD Strong drinkers ( $\geq 3$ unit/day), LD Lower drinkers and minimal oxygen saturation) nocturnal hypoxemia and a higher mean heart rhythm (HR) (Tables 2 and 3).

In multiple regression modeling, diabetes was significantly associated with female sex, increasing age, enlarged $\mathrm{NC}$, high BMI, former or current smoking status, and less alcohol consumption. Severity of OSA (reflected by AHI), decreased sleep quality (reflected by low SE, TST, N3, REM), sleep fragmentation (ARI), nocturnal intermittent/ global hypoxemia, PLM index and a higher mean nocturnal HR were predictors of diabetes. When adjusted for age, BMI, and smoking status, all of these predictors remained significant, and the duration of sleep $\mathrm{AH}$ in REM/NREM also became a determinant factor (Table 4).

In logistic regression, the results showed significant values between REM predominant OSA and diabetes status (Table 5).

\section{Cardiovascular comorbidities (CVCo)}

Comparing patients with CVCo and without, CVCo patients were older, more often women, and non-smokers. They also exhibited worse sleep quality, more marked intermittent/global nocturnal hypoxemia, and a lower mean HR (Tables 2 and 3).

In multiple regression modeling, the presence of $\geq 1$ CVCo was associated with enlarged $\mathrm{NC}$ and alcohol consumption, but not with age, sex, BMI, and smoking status. Severity of OSA, decreased sleep quality, PLM index and nocturnal intermittent/global hypoxemia were predictors of CVCo. When adjusted for age, BMI, dyslipidemia, and smoking status, all of these predictors remained significant (Table 6).

When we evaluated qualitative dependent variables, the logistic regression resulted in non-significant results between CVCo and supine or REM predominant OSA.

\section{Dyslipidemia}

Comparing dyslipidemic and non-dyslipidemic patients, dyslipidemic patients were older, had a larger $\mathrm{NC}$, and were more often drinkers. They also exhibited worse sleep quality and longer AH in REM sleep (Tables 2 and 5).

In multiple regression modeling, the presence of dyslipidemia was associated with enlarged NC, low REM percentage, and longer AH in REM sleep. When adjusted for age, sex, and BMI, dyslipidemia was associated with the preceding factors and the absence of alcohol consumption, OSA severity, decreased sleep quality, PLM index and lower nocturnal HR. (Table 7).

In logistic regression analysis only REM predominant OSA was associated with dyslipidemia (OR: 0.742 [0.579_ $0.950], p=0.018)$.

\section{Discussion}

In this large retrospective study, we have shown that in moderate-to-severe OSA, the presence of cardiometabolic 


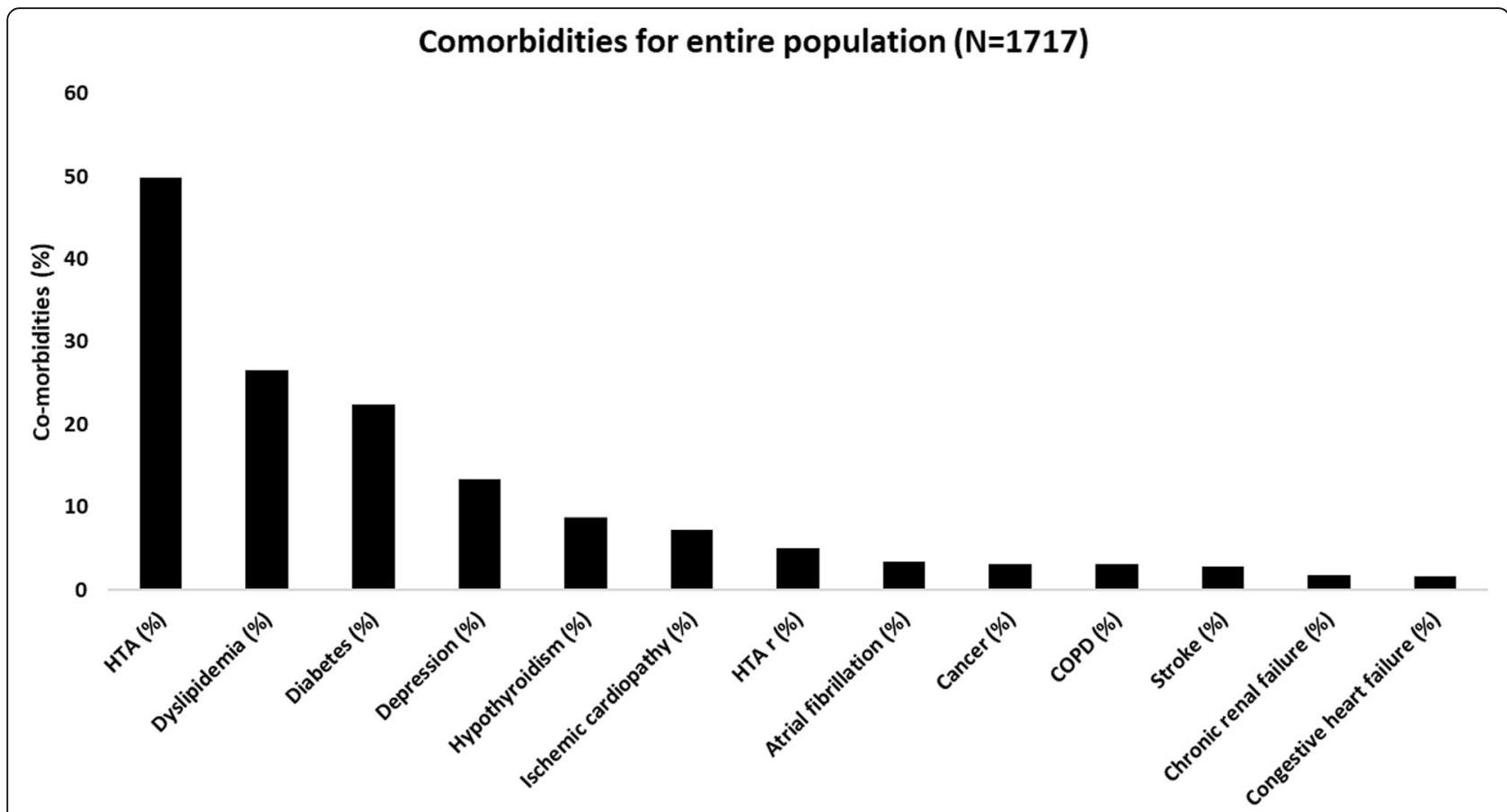

Fig. 1 Comorbidities among included patients. COPD: chronic obstructive pulmonary disease, HTA: hypertension, HTA r: resistant hypertension

complications is associated with a large neck and a particular polysomnographic pattern for diabetes, dyslipidemia, and cardiovascular disease. Indeed, correlations between polysomnographic parameters and the presence of cardiometabolic complications, after adjustment for confounding factors, show that AHI, AH duration, reduced sleep efficiency and total sleep time, low REM proportion, and nocturnal hypoxemia are risk factors associated with all complications. Moreover, absence of alcohol consumption is also associated with metabolic disorders.

OSA is associated with an increased risk of metabolic syndrome [12], including dyslipidemia and insulin resistance, and multiple cardiovascular comorbidities, such as hypertension, coronary artery disease, arrhythmias, ischemic stroke, and congestive heart failure [13, 14]. Is it surprising that all of these comorbidities share the same polysomnographic picture?

Severity of OSA is typically described by the AHI, measuring the number of obstructive events during each hour of sleep. Occurrence of comorbidities increases in parallel with OSA severity.

Indeed, nearly 20 years ago, a linear relationship between AHI and incidental HTA was described by Peppard et al. [15], in a cohort of 709 patients followed for 4 years [15]. Similarly, the risk of stroke or death has been shown to be correlated with OSA severity in a large series of 1022 OSA patients who were followed for 3.4

Table 2 Demographic characteristics for all cardiometabolic comorbidities

\begin{tabular}{|c|c|c|c|c|c|c|c|c|c|}
\hline & $\begin{array}{l}\text { Diabetes } \\
n=386\end{array}$ & $\begin{array}{l}\text { No diabetes } \\
n=1331\end{array}$ & $p$ value & $\begin{array}{l}\text { CVCo } \\
n=671\end{array}$ & $\begin{array}{l}\text { No CVCo } \\
n=1046\end{array}$ & $p$ value & $\begin{array}{l}\text { Dyslipidemia } \\
n=453\end{array}$ & $\begin{array}{l}\text { No dyslipidemia } \\
n=1253\end{array}$ & $p$ value \\
\hline Age $($ mean $\pm S D)$ & $58.33 \pm 11.41$ & $51.23 \pm 12.64$ & 0.0001 & $57.63 \pm 11.42$ & $47.65 \pm 11.98$ & $<0.0001$ & $58.20 \pm 11.49$ & $50.84 \pm 12.56$ & $<0.0001$ \\
\hline Sex (\%) & $\begin{array}{l}F: 37.31 \\
\text { M: } 62.69\end{array}$ & $\begin{array}{l}F: 32.30 \\
M: 67.70\end{array}$ & 0.0023 & $\begin{array}{l}F: 37.74 \\
M: 62.26\end{array}$ & $\begin{array}{l}\text { F: } 28.64 \\
\text { M: } 71.36\end{array}$ & 0.0001 & $\begin{array}{l}F: 33,33 \% \\
H: 66.66 \%\end{array}$ & $\begin{array}{l}F: 33,44 \% \\
H: 66.56 \%\end{array}$ & NS \\
\hline BMI (kg/m2) & $35.52 \pm 7.48$ & $33.70 \pm 8.14$ & 0.0001 & $34.73 \pm 7.59$ & $33.46 \pm 8.41$ & NS & $34.36 \pm 7.46$ & $34.02 \pm 8.21$ & NS \\
\hline $\mathrm{NC}(\mathrm{cm})$ & $43.22 \pm 4.68$ & $42.32 \pm 4.74$ & 0.0011 & $42.75 \pm 4.56$ & $42.30 \pm 4.92$ & NS & $43.03 \pm 4.70$ & $42.34 \pm 4.73$ & 0.0181 \\
\hline Smoking (\%) & $\begin{array}{l}\text { S: } 15.80 \\
\text { NS: } 84.20 \\
\text { EX: } 5.96\end{array}$ & $\begin{array}{l}\text { S: } 24.91 \\
\text { NS: } 75.09 \\
\text { EX: } 3.02\end{array}$ & $<0.0001$ & $\begin{array}{l}\text { S: } 19.15 \\
\text { NS: } 80.85 \\
\text { EX: } 3.81\end{array}$ & $\begin{array}{l}\text { S: } 26.94 \\
\text { NS: } 73.06 \\
\text { EX: } 3.52\end{array}$ & 0.0001 & $\begin{array}{l}\text { S: } 20.31 \\
\text { NS: } 71.52 \\
\text { Ex: } 8.17\end{array}$ & $\begin{array}{l}\text { S: } 23.86 \\
\text { NS: } 71.34 \\
\text { EX: } 4.80\end{array}$ & NS \\
\hline Alcohol (\%) & $\begin{array}{l}\text { ND: } 55.96 \\
\text { StD: } 11.92 \\
\text { LD: } 2.33\end{array}$ & $\begin{array}{l}\text { ND: } 43.09 \\
\text { StD: } 21.96 \\
\text { LD: } 4.15\end{array}$ & 0.0021 & $\begin{array}{l}\text { ND: } 46.36 \\
\text { StD: } 19.15 \\
\text { LD: } 3.58\end{array}$ & $\begin{array}{l}\text { ND: } 45.51 \\
\text { StD: } 20.15 \\
\text { LD: } 3.88\end{array}$ & NS & $\begin{array}{l}\text { ND: } 69,93 \\
\text { StD: } 6.25 \\
\text { LD: } 13.68\end{array}$ & $\begin{array}{l}\text { ND: } 66,26 \\
\text { StD: } 3.19 \text { idem } \\
\text { LD: } 21.94\end{array}$ & 0.0085 \\
\hline
\end{tabular}

Quantitative data are presented with mean and standard deviation and qualitative data as percentages

$B M I$ Body mass index, NC Neck circumference, CVCo Cardiovascular Comorbidities, ND non drinkers, StD Strong drinkers (>3 unit/day), LD Lower drinkers 
Table 3 Polysomnographic characteristics for all cardiometabolic comorbidities

\begin{tabular}{|c|c|c|c|c|c|c|c|c|c|}
\hline & $\begin{array}{l}\text { Diabetes } \\
n=386\end{array}$ & $\begin{array}{l}\text { No diabetes } \\
n=1331\end{array}$ & $p$ value & $\begin{array}{l}\text { CVCo } \\
n=671\end{array}$ & $\begin{array}{l}\text { No CVCo } \\
n=1046\end{array}$ & $p$ value & $\begin{array}{l}\text { Dyslipidemia } \\
n=453\end{array}$ & $\begin{array}{l}\text { No dyslipidemia } \\
n=1253\end{array}$ & $p$ value \\
\hline $\mathrm{AHI}$ & $45.82 \pm 25.77$ & $42.23 \pm 25.60$ & 0.0156 & $43.58 \pm 24.59$ & $42.50 \pm 26.85$ & NS & $44.12 \pm 24.31$ & $42.66 \pm 26.18$ & NS \\
\hline AHI/REM & $50.01 \pm 27.29$ & $48.38 \pm 26.99$ & NS & $49.80 \pm 26.77$ & $47.61 \pm 27.32$ & NS & $47.53 \pm 26.22$ & $49.19 \pm 27.34$ & NS \\
\hline AHI/NREM & $41.73 \pm 26.26$ & $38.46 \pm 26.87$ & 0.0342 & $39.40 \pm 25.48$ & $39.03 \pm 28.13$ & NS & $40.39 \pm 25.19$ & $38.79 \pm 27.33$ & NS \\
\hline$S L(\min )$ & $70.01 \pm 62.26$ & $73.20 \pm 62.06$ & NS & $70.05 \pm 59.99$ & $75.20 \pm 64.20$ & NS & $68.20 \pm 60.82$ & $74.11 \pm 62.48$ & NS \\
\hline SE (\%) & $63.61 \pm 15.05$ & $67.42 \pm 14.57$ & 0.0001 & $64.89 \pm 14.94$ & $68.30 \pm 14.45$ & 0.0001 & $64.65 \pm 14.65$ & $67.21 \pm 14.80$ & 0.0022 \\
\hline TST (min) & $327.12 \pm 78.35$ & $343.64 \pm 76.60$ & 0.0002 & $331.59 \pm 79.02$ & $348.56 \pm 74.81$ & 0.0001 & $330.79 \pm 79.06$ & $343.04 \pm 76.64$ & 0.0052 \\
\hline N1 (\%) & $4.55 \pm 4.56$ & $4.13 \pm 3.62$ & NS & $4.56 \pm 4.27$ & $3.90 \pm 3.39$ & 0.0156 & $4.92 \pm 4.74$ & $3.99 \pm 3.48$ & $<0.0001$ \\
\hline N2 (\%) & $50.88 \pm 16.37$ & $50.36 \pm 15.89$ & NS & $50.61 \pm 15.50$ & $50.40 \pm 16.50$ & NS & $51.27 \pm 15.81$ & $50.22 \pm 16.03$ & NS \\
\hline N3 (\%) & $29.11 \pm 17.19$ & $28.67 \pm 15.79$ & NS & $28.80 \pm 15.81$ & $28.69 \pm 16.41$ & NS & $27.77 \pm 16.28$ & $29.10 \pm 16.01$ & NS \\
\hline REM (\%) & $15.52 \pm 7.32$ & $16.91 \pm 6.88$ & 0.0006 & $16.16 \pm 7.29$ & $17.04 \pm 6.65$ & 0.0091 & $16.17 \pm 7.34$ & $16.72 \pm 7.34$ & NS \\
\hline ARI & $39.91 \pm 18.34$ & $37.13 \pm 17.94$ & 0.0007 & $37.91 \pm 17.04$ & $37.67 \pm 19.14$ & NS & $39.39 \pm 17.55$ & $37.20 \pm 17.55$ & 0.0356 \\
\hline $\begin{array}{l}\text { Mean duration } \\
\text { AH REM (sec) }\end{array}$ & $25.59 \pm 11.00$ & $26.36 \pm 9.69$ & NS & $26.46 \pm 10.41$ & $25.89 \pm 9.56$ & NS & $27.09 \pm 11.68$ & $25.83 \pm 11.68$ & 0.0429 \\
\hline $\begin{array}{l}\text { Mean duration } \\
\text { AH NREM (sec) }\end{array}$ & $21.90 \pm 6.02$ & $22.41 \pm 5.96$ & NS & $22.32 \pm 5.73$ & $22.24 \pm 6.24$ & NS & $22.63 \pm 6.17$ & $22.15 \pm 6.17$ & NS \\
\hline PLM & $6.31 \pm 22.47$ & $5.63 \pm 16.80$ & NS & $6.23 \pm 18.97$ & $5.26 \pm 17.33$ & NS & $5.87 \pm 19.18$ & $5.73 \pm 17.83$ & NS \\
\hline ODI & $57.42 \pm 32.30$ & $45.37 \pm 30.60$ & $<0.0001$ & $50.44 \pm 30.42$ & $45.79 \pm 32.36$ & 0.0357 & $50.28 \pm 31.87$ & $47.60 \pm 31.87$ & NS \\
\hline $\begin{array}{l}\text { Mean } 02 \\
\text { saturation } \\
\text { TST (\%) }\end{array}$ & $92.81 \pm 30.94$ & $92.18 \pm 2.67$ & NS & $92.27 \pm 20.47$ & $92.35 \pm 2.75$ & NS & $91.54 \pm 2.88$ & $92.59 \pm 2.88$ & NS \\
\hline $\begin{array}{l}02 \\
\text { desaturation } \\
<90 \% \text { (min) }\end{array}$ & $102.33 \pm 115.04$ & $74.44 \pm 99.13$ & $<0.0001$ & $91.67 \pm 108.71$ & $69.54 \pm 97.24$ & $\begin{array}{l}< \\
0.0001\end{array}$ & $96.06 \pm 113.31$ & $75.47 \pm 113.31$ & 0.0005 \\
\hline $\begin{array}{l}\text { Minimal } 02 \\
\text { saturation (\%) }\end{array}$ & $75.14 \pm 10.42$ & $77.58 \pm 9.41$ & $<0.0001$ & $76.43 \pm 9.64$ & $77.62 \pm 9.83$ & 0.0203 & $76.20 \pm 9.78$ & $77.28 \pm 9.78$ & NS \\
\hline $\begin{array}{l}\text { Mean HR } \\
\text { (beats/min) }\end{array}$ & $70.78 \pm 10.36$ & $68.10 \pm 9.95$ & $<0.0001$ & $67.88 \pm 10.25$ & $69.63 \pm 9.85$ & 0.0003 & $68.20 \pm 10.20$ & $69.91 \pm 10.20$ & NS \\
\hline
\end{tabular}

AHI Apnea-hypopnea index, AH Apnea-hypopnea, REM Rapid eye movement sleep, NREM Non-rapid eye movement sleep, CVCo Cardiovascular Comorbidities, SL Sleep latency, TST Total sleep time, SE Sleep efficiency, N1 Sleep stage 1, N2 Sleep stage 2, N3 Sleep stage 3, PLM Periodic leg movements index, ODI Oxygen desaturation index, ARI Arousal index, 02 Oxygen, HR: heart rhythm. All the results are expressed as Mean \pm SD. NS: not significant

years [16]. Oxidative stress, endothelial dysfunction, systemic inflammation, and activation of the sympathetic nervous system are the pathophysiological mechanisms leading to cardiovascular disorders in OSA, as a consequence of repeated obstructive sleep apnea and hypopnea, inducing hypoxia/hypercapnia, arousals, and negative intra-thoracic pressure [17].

Ip et al. have shown that insulin resistance is independently associated with increasing AHI [18]. In untreated diabetic patients, HbA1c levels have been shown to be positively associated with AHI [19]. Recent data from the European ESADA cohort have shown that the prevalence of diabetes increases with OSA severity, reaching $29 \%$ in patients with severe OSA [20]. Underlying mechanisms include intermittent hypoxia and sleep fragmentation, activation of the sympathetic nervous system, oxidative stress, systemic inflammation, alterations in appetite-regulating hormones, and activation of the hypothalamic-pituitary-adrenal axis which leads first to the development of insulin resistance and then progresses to glucose intolerance and diabetes [21]. Guan et al., in an observational cohort, have also shown a linear dose effect between AHI and dyslipidemia [22]. Altogether, our results confirm this strong association between OSA severity, reflected by AHI, with the presence of cardiometabolic comorbidities that share the same pathophysiological mechanisms.

However, in clinical practice, we know that the widely used AHI is not sufficiently accurate to describe the full picture of OSA. In the present study, we have highlighted that mean duration of AH in REM and nonREM sleep is also an independent predictor of the presence of cardiometabolic comorbidities. Wu et al., have shown, in a retrospective study, that a longer mean $\mathrm{AH}$ duration (but not $\mathrm{AHI}$ ) is associated with higher odds of moderate-to-severe HTA [23]. Therefore, clinicians should be advised to pay attention not only to AHI but also to HA length in order to assess OSA severity.

The same group has shown, in a rat model, that longer $\mathrm{AH}$ duration is associated with more systemic 
Table 4 Linear regression analysis for the influence of diabetes-independent variables on quantitative dependent variables

\begin{tabular}{|c|c|c|c|c|c|c|c|}
\hline & \multirow[b]{2}{*}{ Dependent variables } & \multicolumn{3}{|c|}{ Model 1: diabetes } & \multicolumn{3}{|c|}{ Model 2: diabetes + adjustement (age, BMI, smoking) } \\
\hline & & $\bar{R}$ & $\mathrm{R}^{2}$ & $\overline{P \text { value }}$ & $\bar{R}$ & $R^{2}$ & $P$ value \\
\hline \multirow[t]{2}{*}{ Demographic data } & NC & 0.091 & 0.008 & $<0.0001$ & 0.434 & 0.186 & $<0.0001$ \\
\hline & Alcohol & 0.089 & 0.007 & 0.002 & 0.224 & 0.047 & $<0.0001$ \\
\hline \multirow[t]{16}{*}{ Polysomnographic data } & $\mathrm{AHI}$ & 0.060 & 0.003 & 0.015 & 0.287 & 0.080 & $<0.0001$ \\
\hline & $\mathrm{AHI} / \mathrm{REM}$ & 0.029 & 0.000 & NS & 0.345 & 0.117 & $<0.0001$ \\
\hline & $\mathrm{AHI} / \mathrm{NREM}$ & 0.053 & 0.002 & 0.031 & 0.260 & 0.066 & $<0.0001$ \\
\hline & SE (\%) & 0.111 & 0.012 & $<0.0001$ & 0.204 & 0.039 & 0.0001 \\
\hline & TST (min) & 0.093 & 0.008 & $<0.0001$ & 0.189 & 0.033 & $<0.0001$ \\
\hline & N3 (\%) & 0.006 & -0.001 & NS & 0.124 & 0.013 & $<0.0001$ \\
\hline & REM (\%) & 0.089 & 0.007 & $<0.0001$ & 0.169 & 0.026 & $<0.0001$ \\
\hline & ARI & 0.068 & 0.001 & 0.005 & 0.179 & 0.030 & $<0.0001$ \\
\hline & $\begin{array}{l}\text { Mean duration } \\
\text { AH REM (sec) }\end{array}$ & 0.043 & 0.001 & NS & 0.202 & 0.038 & $<0.0001$ \\
\hline & $\begin{array}{l}\text { Mean duration } \\
\text { AH NREM (sec) }\end{array}$ & 0.044 & 0.001 & NS & 0.303 & 0.090 & $<0.0001$ \\
\hline & ODI & 0.167 & 0.027 & $<0.0001$ & 0.364 & 0.128 & $<0.0001$ \\
\hline & PLM & 0.013 & $<0.0001$ & NS & 0.141 & 0.018 & $<0.0001$ \\
\hline & $\begin{array}{l}\text { Mean } 02 \text { saturation } \\
\text { TST (\%) }\end{array}$ & 0.019 & 0.000 & NS & 0.130 & 0.014 & $<0.0001$ \\
\hline & $\begin{array}{l}02 \text { desaturation } \\
<90 \% \text { (min) }\end{array}$ & 0.109 & 0.011 & $<0.0001$ & 0.341 & 0.114 & $<0.0001$ \\
\hline & $\begin{array}{l}\text { Minimal } 02 \\
\text { saturation (\%) }\end{array}$ & 0.111 & 0.012 & $<0.0001$ & 0.282 & 0.077 & $<0.0001$ \\
\hline & Mean HR (beats/min) & 0.108 & 0.011 & $<0.0001$ & 0.373 & 0.137 & $<0.0001$ \\
\hline
\end{tabular}

BMI Body mass index, NC Neck circumference, AHI Apnea-hypopnea index, AH Apnea-hypopnea, REM Rapid eye movement sleep, NREM Non-rapid eye movement sleep, SL Sleep latency, TST Total sleep time, SE Sleep efficiency, N3 Sleep stage 3, PLM Periodic leg movements index, ODI Oxygen desaturation index, ARI Arousal index, $H R$ Heart rhythm, $O_{2}$ Oxygen. All the results are expressed as Mean + SD. NS Not significant

inflammatory and endothelial dysfunction, that can (partially) recover when duration decreases or when sleep apneas are suppressed [24].

It is interesting to note that heart rate excursion after an obstructive apnea is associated with apnea duration and not with AHI [25]. Longer apnea duration also implies deeper desaturation, which has been shown to be associated with a higher risk of atrial fibrillation [26].

Table $\mathbf{5}$ Logistic regression analysis for the influence of diabetes-independent variables on qualitative dependent variables, adjusted for age, BMI, and smoking status

\begin{tabular}{lllll}
\hline & Variables & $\begin{array}{l}\text { Odd } \\
\text { ratio }\end{array}$ & $\begin{array}{l}95 \% \text { confidence } \\
\text { interval }\end{array}$ & $P$ value \\
\hline Demographic & Sex & 0.891 & $0.688-1.155$ & NS \\
Polysomnography & $\begin{array}{l}\text { Supine } \\
\text { predominant }\end{array}$ & 0.990 & $0.683-1.153$ & NS \\
& $\begin{array}{l}\text { OSA } \\
\text { REM }\end{array}$ & 0.712 & $0.548-0.926$ & 0.011 \\
& $\begin{array}{l}\text { Rredominant } \\
\text { OSA }\end{array}$ & & & \\
\hline
\end{tabular}

REM Rapid eye movement sleep, OSA Obstructive sleep apnea syndrome
Future prospective studies are needed to discern the specific impact of AHI and of the mean duration of $\mathrm{AH}$ on comorbidities and symptoms.

We have also to stress that if one wants to use $\mathrm{AH}$ duration as a metric in the future, it is mandatory to rely on manually scored AH according to AASM scoring rules [9] and not to automated measurements made by the PSG systems, because the AH detection algorithms can vary from one device to another.In this study, poor sleep was also associated with the presence of cardiometabolic comorbidities.

Independently from OSA, poor sleep (increased sleep latency, wake after sleep onset, reduced SE, TST, N3, and REM sleep) have been demonstrated as risk factors for diabetes $[27,28]$, with $13-21 \%$ of insomniacs affected by the disorder. Patients suffering from insomnia and diabetes have more comorbidities (e.g. HTA, dyslipidemia), are generally overweight/obese, and have a short sleep duration, from less than $390 \mathrm{~min}$ [28]. Insomnia could also help identifying more severe patients. Indeed, it has been shown, in elderly community-dwelling adults, that comorbid insomnia was related to hypoxemia in 
Table 6 Linear regression analysis for the influence of multiple cardiac-independent variables (CVCo) on quantitative dependent variables

\begin{tabular}{|c|c|c|c|c|c|c|c|}
\hline & \multirow[b]{2}{*}{ Dependent variables } & \multicolumn{3}{|c|}{ Model 1: CVCo } & \multicolumn{3}{|c|}{ Model 2: CVCo + adjustement (age, BMI, smoking, sex, dyslipidemia) } \\
\hline & & $\bar{R}$ & $R^{2}$ & $P$ value & $R$ & $\mathrm{R}^{2}$ & $P$ value \\
\hline \multirow[t]{2}{*}{ Demographic data } & NC & 0.092 & 0.007 & 0.001 & 0.722 & 0.519 & $<0.0001$ \\
\hline & Alcool & 0.095 & 0.007 & 0.005 & 0.263 & 0.065 & $<0.0001$ \\
\hline \multirow[t]{16}{*}{ Polysomnographic data } & $\mathrm{AHI}$ & 0.060 & 0.002 & 0.049 & 0.352 & 0.120 & $<0.0001$ \\
\hline & $\mathrm{AHI} / \mathrm{REM}$ & 0.064 & 0.003 & 0.034 & 0.348 & 0.118 & $<0.0001$ \\
\hline & $\mathrm{AHI} / \mathrm{NREM}$ & 0.054 & 0.002 & NS & 0.341 & 0.112 & $<0.0001$ \\
\hline & SE (\%) & 0.064 & 0.003 & 0.047 & 0.268 & 0.067 & $<0.0001$ \\
\hline & TST (min) & 0.079 & 0.005 & 0.010 & 0.376 & 0.137 & $<0.0001$ \\
\hline & N3 (\%) & 0.023 & 0.001 & NS & 0.074 & 0.001 & NS \\
\hline & REM (\%) & 0.130 & 0.016 & $<0.0001$ & 0.215 & 0.042 & $<0.0001$ \\
\hline & ARI & 0.120 & 0.013 & $<0.0001$ & 0.196 & 0.034 & $<0.0001$ \\
\hline & $\begin{array}{l}\text { Mean duration } \\
\text { AH REM (sec) }\end{array}$ & 0.092 & 0.007 & 0.001 & 0.200 & 0.036 & $<0.0001$ \\
\hline & $\begin{array}{l}\text { Mean duration } \\
\text { AH NREM (sec) }\end{array}$ & 0.021 & 0.001 & NS & 0.217 & 0.043 & $<0.0001$ \\
\hline & PLM & 0.027 & $<0.0001$ & NS & 0.142 & 0.018 & $<0.0001$ \\
\hline & ODI (h) & 0.009 & 0.001 & NS & 0.250 & 0.059 & $<0.0001$ \\
\hline & $\begin{array}{l}\text { Mean } 02 \text { saturation } \\
\text { TST (\%) }\end{array}$ & 0.101 & 0.009 & $<0.0001$ & 0.176 & 0.027 & $<0.0001$ \\
\hline & $\begin{array}{l}02 \text { desaturation } \\
<90 \% \text { (min) }\end{array}$ & 0.067 & 0.003 & 0.024 & 0.261 & 0.064 & $<0.0001$ \\
\hline & $\begin{array}{l}\text { Minimal } 02 \\
\text { saturation (\%) }\end{array}$ & 0.092 & 0.007 & 0.001 & 0.283 & 0.076 & $<0.0001$ \\
\hline & Mean HR (beats/min) & 0.121 & 0.014 & $<0.0001$ & 0.363 & 0.128 & $<0.0001$ \\
\hline
\end{tabular}

CVCo Cardiovascular Comorbidities, BMI Body mass index, NC Neck circumference, AHI Apnea-hypopnea index, AH Apnea-hypopnea, REM Rapid eye movement sleep, NREM Non-rapid eye movement sleep, SL Sleep latency, TST Total sleep time, SE Sleep efficiency, N3 Sleep stage 3, PLM Periodic leg movements index, ODI Oxygen desaturation index, $A R I$ Arousal index, $\mathrm{O}_{2}$ Oxygen, $H R$ Heart rhythm. All the results are expressed as Mean \pm SD. NS Not significant

OSA patients suffering from cardiovascular disease, and associated to a worse prognosis [29].

In our study, polysomnographic markers of decreased sleep quality (reduced SE, TST, N3, and REM sleep, and increased ARI) were correlated with the presence of cardiometabolic comorbidities, and may be a consequence of OSA or an associated primary insomnia, which occurs frequently in OSA, in about $44-55 \%$ of patients [30]. This comorbid insomnia has a negative additive effect on comorbidities through the activation of the hypothalamicpituitary-adrenal axis and excessive sympathetic nervous activity [28].

Sleep fragmentation, a typical pattern of OSA, is associated with decreased insulin sensitivity in healthy subjects, through increases in sympathetic nervous system and adrenocortical activity [31] and can aggravate the metabolic effects of short sleep duration in OSA patients.

Short sleep duration, less than $6 \mathrm{~h} /$ night, was also shown to be associated with diabetes incidence but not with incidental cardiovascular disease in a very large meta-analysis [32]. Underlying pathophysiological mechanisms include decreased glucose tolerance and insulin sensitivity, increased evening concentrations of cortisol and levels of ghrelin, decreased levels of leptin, and increased hunger and appetite [33]. Prolonged short sleep durations could also lead to hypertension through raised 24-h blood pressure and increased salt retention [34].

PLM severity was also associated with cardiometabolic disorders. It has been documented that PLM induces blood pressure elevations and heart rate elevations [35]. These two markers are now recognized as surrogates for cardiovascular risk, such that our findings are not surprising. Yet, no data have been published regarding the association of PLM and metabolic disorders. There are however published data regarding the impact of sleep fragmentation on glucose metabolism disorders in humans [21] and on dyslipidemia in mice [36]. As PLM induce sleep fragmentation [37, 38], it seems to be the most plausible underlying physiopathological mechanisms.

Finally, nocturnal hypoxemia was invariably correlated with the presence of cardiometabolic comorbidities.

Intermittent hypoxia is the hallmark of OSA. It is characterized by repetitive profound hypoxic periods followed 
Table 7 Linear regression analysis for the influence of dyslipidemia-independent variables on quantitative dependent variables

\begin{tabular}{|c|c|c|c|c|c|c|c|}
\hline & \multirow[b]{2}{*}{ Dependent variables } & \multicolumn{3}{|c|}{ Model 1: Dyslipidemia } & \multicolumn{3}{|c|}{ Model 2: Diyslipidemia + adjustement (age, BMI, sex) } \\
\hline & & $\mathrm{R}$ & $\mathrm{R}^{2}$ & $P$ value & $\bar{R}$ & $\mathrm{R}^{2}$ & $P$ value \\
\hline \multirow[t]{2}{*}{ Demographic data } & NC & 0.058 & 0.003 & 0.018 & 0.710 & 0.502 & $<0.0001$ \\
\hline & Alcool & 0.032 & 0.000 & NS & 0.190 & 0.033 & $<0.0001$ \\
\hline \multirow[t]{16}{*}{ Polysomnographic data } & $\mathrm{AHI}$ & 0.023 & 0.000 & NS & 0.351 & 0.121 & $<0.0001$ \\
\hline & $\mathrm{AHI} / \mathrm{REM}$ & 0.027 & 0.000 & NS & 0.353 & 0.123 & $<0.0001$ \\
\hline & $\mathrm{AHI} / \mathrm{NREM}$ & 0.023 & 0.000 & NS & 0.338 & 0.112 & $<0.0001$ \\
\hline & SE (\%) & 0.036 & 0.001 & NS & 0.251 & 0.061 & $<0.0001$ \\
\hline & TST (min) & 0.018 & 0.000 & NS & 0.378 & 0.141 & $<0.0001$ \\
\hline & N3 (\%) & 0.043 & 0.001 & NS & 0.068 & 0.002 & NS \\
\hline & REM (\%) & 0.074 & 0.005 & 0.002 & 0.204 & 0.039 & $<0.0001$ \\
\hline & ARI & 0.067 & 0.004 & 0.005 & 0.182 & 0.031 & $<0.0001$ \\
\hline & $\begin{array}{l}\text { Mean duration } \\
\text { AH REM (sec) }\end{array}$ & 0.104 & 0.010 & $<0.0001$ & 0.182 & 0.031 & $<0.0001$ \\
\hline & $\begin{array}{l}\text { Mean duration } \\
\text { AH NREM (sec) }\end{array}$ & 0.027 & 0.000 & NS & 0.200 & 0.038 & $<0.0001$ \\
\hline & PLM & 0.001 & 0.001 & NS & 0.139 & 0.017 & $<0.0001$ \\
\hline & ODI & 0.035 & 0.001 & NS & 0.229 & 0.050 & $<0.0001$ \\
\hline & $\begin{array}{l}\text { Mean } 02 \text { saturation } \\
\text { TST (\%) }\end{array}$ & 0.033 & 0.000 & NS & 0.153 & 0.024 & $<0.0001$ \\
\hline & $\begin{array}{l}02 \text { desaturation } \\
<90 \% \text { (min) }\end{array}$ & 0.051 & 0.002 & 0.036 & 0.252 & 0.061 & $<0.0001$ \\
\hline & $\begin{array}{l}\text { Minimal } 02 \\
\text { saturation (\%) }\end{array}$ & 0.054 & 0.002 & 0.030 & 0.277 & 0.074 & $<0.0001$ \\
\hline & Mean HR (beats/min) & 0.035 & 0.001 & NS & 0.353 & 0.123 & $<0.0001$ \\
\hline
\end{tabular}

BMI: body mass index, NC: neck circumference, AHI: apnea-hypopnea index, AH: apnea-hypopnea, REM: rapid eye movement sleep, NREM: non-rapid eye movement sleep, SL: sleep latency, TST: total sleep time, SE: sleep efficiency, N3: sleep stage 3, PLM: periodic leg movements index, ODI: oxygen desaturation index, ARI: arousal index, $\mathrm{O}_{2}$ : oxygen, HR: heart rhythm. All the results are expressed as Mean \pm SD. NS: not significant

by blood reoxygenation. It has been hypothesized that these phenomena are similar to repeated ischemia and reperfusion events which result in release of reactive oxygen species and activate oxidative stress pathways, promoting cardiovascular morbidity in OSA [39].

OSA seems also to promote post-prandial hyperlipidemia through intermittent hypoxia. It has been demonstrated first in a mouse model [40], then in humans by Phillips et al. [41], showing that in humans with OSA, post-prandial lipids are elevated and favorably impacted by CPAP [41]. The benefit of CPAP on total cholesterol levels was confirmed in a recent meta-analysis [42].

Intermittent hypoxia seems also to play a role in glucose metabolism disorders. Indeed, Tassone et al. have compared obese and OSA obese patients and have shown that OSA obese patients exhibit more insulin resistance, independently of fat distribution, suggesting a role of intermittent hypoxia [43].

In our retrospective series, patients suffering from cardiometabolic comorbidities also had a larger NC than their healthier OSA counterparts. Even after adjustment for BMI, enlarged NC remained a risk factor. Fat accumulation in the neck is a sign of visceral obesity, responsible for OSA and the metabolic syndrome [12], and an additional factor promoting cardiovascular comorbidities [44].

Our last finding regards alcohol consumption. There were more metabolic comorbidities in non-drinkers and more cardiovascular disorders in drinkers. Recent data on the metabolic syndrome have confirmed a lower prevalence in patients who eat seafood and non-starchy vegetables, and drink moderate amounts of alcohol [45], in concordance with our findings. Moderate alcohol consumption has been shown to increase insulin sensitivity [46], and to contributes to a lower risk of diabetes development in Japanese men [47]. It leads also to lower HDLc levels and adiponectin, which inhibits liver fat accumulation [48]. Regarding cardiovascular disorders, the negative effect of alcohol is dose-dependent and related to the type of ingested alcohol $[49,50]$, and it is thus not surprising to see a negative effect of alcohol in our population.

\section{Limitations}

The results of this study come from retrospective data analysis. This design could potentially lead to missing data or biases. Moreover, in the absence of control group (patients without OSA), it is difficult to ascertain 
the associations between sleep parameters and cardiometabolic comorbidities. Thus, our results should be confirmed in prospective/controlled series.

\section{Conclusions}

This study identifies particular demographic and polysomnographic factors associated with cardiometabolic comorbidities. Indeed, patients (especially women) suffering from more severe OSA, longer sleep apneas and hypopneas, worse sleep quality, and marked intermittent/global nocturnal hypoxemia are more likely to develop cardiometabolic comorbidities. Particular attention should be paid to all these parameters when choosing OSA treatment on an individual basis. This should also stimulate clinicians to obtain and maintain adequate treatment in this population, in order to prevent future complications. Moreover, further studies need to be conducted, prospectively, to identify the added value of comorbid insomnia in the occurrence of cardiometabolic comorbidities in OSA.

\section{Abbreviations \\ 02: Oxygen; AH: Apnea- hypopnea; AHI: Apnea- hypopnea index; ARI: Arousal index; BMI: Body Mass Index; CPAP: Continuous positive airway pressure; CVCo: Cardiovascular comorbidity; HR: Heart rhythm; HTA: Hypertension; N1: sleep stage 1; N2: sleep stage 2; N3: sleep stage 3; NC: Neck Circumference; NREM: Non- Rapid Eye Movement; ODI: Oxygen Desaturation Index; OSA: Obstructive Sleep Apnea Syndrome; PLM: Periodic Leg Movements; PSG: Polysomnography; REM: Rapid Eye Movement; SE: Sleep Efficiency; TST: Total Sleep Time}

\section{Acknowledgements}

The authors would like to acknowledge the contribution of a medical writer, Sandy Field, PhD, for editing of this manuscript.

\section{Authors' contributions}

FA, CVC, SBY, IB, SC, AG, AVB, MB collected the data; SA, FA, AVB, MB performed data analyses and prepared the manuscript. FA, CVC, SBY, IB, SC, $A G, A V B, M B$ have approved the final version of the manuscript.

\section{Funding}

This research did not receive any specific grant from funding agencies in the public, commercial, or not-for-profit sectors.

\section{Availability of data and materials}

The datasets used and/or analysed during the current study are available from the corresponding author on reasonable request.

\section{Ethics approval and consent to participate}

The study protocol was approved by the Saint-Pierre University Hospital ethics committee (CE/18-01-06).

\section{Consent for publication}

Not applicable.

\section{Competing interests}

The authors declare that they have no competing interests.

\section{Author details}

${ }^{1}$ Department of Pulmonary Medicine, Saint-Pierre University Hospital, Rue Haute 322, 1000 Brussels, Belgium. ² nniversité Libre de Bruxelles, Brussels, Belgium. ${ }^{3}$ Department of Pulmonary Medicine, Brugmann University Hospital, Brussels, Belgium. ${ }^{4}$ KiCarre, research department, Lentilly, France.
Received: 7 July 2019 Accepted: 18 October 2019

Published online: 29 January 2020

\section{References}

1. Heinzer R, Vat S, Marques-Vidal P, et al. Prevalence of sleep-disordered breathing in the general population: the HypnoLaus study. Lancet Respir Med. 2015:3(4):310-8.

2. Marin JM, Carrizo SJ, Vicente E, Agusti AG. Long-term cardiovascular outcomes in men with obstructive sleep apnoea-hypopnoea with or without treatment with continuous positive airway pressure: an observational study. Lancet. 2005;365:1046-53.

3. Arzt M, Oldenburg O, Graml A, Erdmann E, Teschler H, Wegscheider K, et al. Phenotyping of Sleep-Disordered Breathing in Patients With Chronic Heart Failure With Reduced Ejection Fraction-the SchlaHF Registry. J Am Heart Assoc. 2017;6:12

4. Mehra R, Stone KL, Varosy PD, Hoffman AR, Marcus GM, Blackwell T, et al. Nocturnal arrhythmias across a spectrum of obstructive and central sleepdisordered breathing in older men: outcomes of sleep disorders in older men (MrOS sleep) study. Arch Intern Med. 2009;169(12):1147-55.

5. Tamura A, Kawano Y, Watanabe T, Kadota J. Relationship between the severity of obstructive sleep apnea and impaired glucose metabolism in patients with obstructive sleep apnea. Respir Med. 2008;102:1412-6.

6. Lam JCM, Lui MMS, Ip MSM. Diabetes and metabolic aspects of OSA. Eur Respi Monogr. 2010;1:189-215.

7. Bailly S, Destors M, Grillet Y, Richard P, Stach B, Vivodtzev I, et al. Obstructive sleep apnea: a cluster analysis at time of diagnosis. PLoS One. 2016;11(6): e0157318

8. Gagnadoux F, Le Vaillant M, Paris A, Pigeanne T, Leclair-Visonneau L, BizieuxThaminy A, et al. Relationship between OSA clinical phenotypes and CPAP treatment outcomes. Chest. 2016;149(1):288-90.

9. Berry RB, Budhiraja R, Gottlieb DJ, Gozal D, Iber C, Kapur VK, et al. Rules for scoring respiratory events in sleep: update of the 2007 AASM manual for the scoring of sleep and associated events. J Clin Sleep Med. 2012;8:597-619.

10. Cartwright RD. Effect of sleep position on sleep apnea severity. Sleep. 1984; 7(2):110-4.

11. Duce B, Kulkas A, Langton C, Toyras J, Hukins C. The prevalence of REMrelated obstructive sleep apnoea is reduced by the AASM 2012 hypopnoea criteria. Sleep Breath. 2018;22(1):57-64.

12. Gaines J, Vgontzas AN, Fernandez-Mendoza J, Bixler EO. Obstructive sleep apnea and the metabolic syndrome: the road to clinically-meaningful phenotyping, improved prognosis, and personalized treatment. Sleep Med Rev. 2018;42:211-9.

13. Bonsignore MR, Baiamonte P, Mazzuca E, Castrogiovanni A, Marrone O. Obstructive sleep apnea and comorbidities: a dangerous liaison. Multidiscip Respir Med. 2019;14:8.

14. Dharia SM, Brown LK. Epidemiology of sleep-disordered breathing and heart failure: what drives what. Curr Heart Fail Rep. 2017;14(5):351-64.

15. Peppard PE, Young T, Barnet JH, Palta M, Hagen EW, Hla KM. Increased prevalence of sleep-disordered breathing in adults. Am J Epidemiol. 2013; 177(9):1006-14.

16. Yaggi HK, Concato J, Kernan WN, Lichtman JH, Brass LM, Mohsenin V. Obstructive sleep apnea as a risk factor for stroke and death. N Engl J Med. 2005;353(19):2034-41.

17. Bradley TD, Floras JS. Obstructive sleep apnoea and its cardiovascular consequences. Lancet. 2009;373(9657):82-93.

18. Ip MS, Lam B, Ng MM, Lam WK, Tsang KW, Lam KS. Obstructive sleep apnea is independently associated with insulin resistance. Am J Respir Crit Care Med. 2002;165:670e6.

19. Priou P, Le Vaillant M, Meslier N, Chollet S, Masson P, Humeau MP, et al. Independent association between obstructive sleep apnea severity and glycated hemoglobin in adults without diabetes. Diabetes Care. 2012;35(9):1902-6.

20. Kent BD, Grote L, Bonsignore MR, Saaresranta T, Verbraecken J, Levy P, et al. Sleep apnoea severity independently predicts glycaemic health in nondiabetic subjects: the ESADA study. Eur Respir J. 2014;44(1):130-9.

21. Martinez Ceron E, Casitas Mateos R, Garcia-Rio F. Sleep apnea-hypopnea syndrome and type 2 diabetes. A reciprocal relationship? Arch Bronconeumol. 2015:51(3):128-39.

22. Guan J, Yi H, Zou J, Meng L, Tang X, Zhu H, et al. Distinct severity stages of obstructive sleep apnoea are correlated with unique dyslipidaemia: largescale observational study. Thorax. 2016;71(4):347-55. 
23. Wu H, Zhan X, Zhao M, Wei Y. Mean apnea-hypopnea duration (but not apnea-hypopnea index) is associated with worse hypertension in patients with obstructive sleep apnea. Medicine (Baltimore). 2016;95(48):e5493.

24. Wu H, Lv Q, Zhang $H$, Qin $Y$, Fang $F$, Sun $H$, et al. The reduction of apneahypopnea duration ameliorates endothelial dysfunction, vascular inflammation, and systemic hypertension in a rat model of obstructive sleep apnea. Sleep Breath. 2019;1:1

25. Sola-Soler J, Giraldo BF, Fiz JA, Jane R. Relationship between heart rate excursion and apnea duration in patients with obstructive sleep apnea. Conf Proc IEEE Eng Med Biol Soc. 2017;2017:1539-42.

26. Gami AS, Hodge DO, Herges RM, Olson EJ, Nykodym J, Kara T, et al. Obstructive sleep apnea, obesity, and the risk of incident atrial fibrillation. J Am Coll Cardiol. 2007;49:565-71.

27. Taylor DJ, Mallory $\sqcup$, Lichstein KL, Durrence HH, Riedel BW, Bush AJ. Comorbidity of chronic insomnia with medical problems. Sleep. 2007;30(2):213-8.

28. Hein M, Lanquart JP, Loas G, Hubain P, Linkowski P. Prevalence and risk factors of type 2 diabetes in insomnia sufferers: a study on 1311 individuals referred for sleep examinations. Sleep Med. 2018;46:37-45.

29. Johansson P, Svensson E, Alehagen U, Jaarsma T, Broström A. The contribution of hypoxia to the association between sleep apnoea, insomnia, and cardiovascular mortality in community-dwelling elderly with and without cardiovascular disease. Eur J Cardiovasc Nurs. 2015;14(3):222-31.

30. Bjorvatn B, Lehmann S, Gulati S, Aurlien H, Pallesen S, Saxvig IW. Prevalence of excessive sleepiness is higher whereas insomnia is lower with greater severity of obstructive sleep apnea. Sleep Breath. 2015;19(4):1387-93.

31. Stamatakis KA, Punjabi NM. Effects of sleep fragmentation on glucose metabolism in normal subjects. Chest. 2010;137(1):95-101.

32. Holliday EG, Magee CA, Kritharides L, Banks E, Attia J. Short sleep duration is associated with risk of future diabetes but not cardiovascular disease: a prospective study and meta-analysis. PLoS One. 2013;8(11):e82305.

33. Leproult $\mathrm{R}$, Van Cauter E. Role of sleep and sleep loss in hormonal release and metabolism. Endocr Dev. 2010;17:11-21.

34. Gangwisch JE. Epidemiological evidence for the links between sleep, circadian rhythms and metabolism. Obes Rev. 2009;10(Suppl 2):37-45.

35. Cassel W, Kesper K, Bauer A, Grieger F, Schollmayer E, Joeres L, et al. Significant association between systolic and diastolic blood pressure elevations and periodic limb movements in patients with idiopathic restless legs syndrome. Sleep Med. 2016;17:109-20.

36. Khalyfa A, Carreras A, Almendros I, Hakim F, Gozal D. Sex dimorphism in late gestational sleep fragmentation and metabolic dysfunction in offspring mice. Sleep. 2015;38:545-57.

37. Sieminski M, Pyrzowski J, Partinen M. Periodic limb movements in sleep are followed by increases in EEG activity, blood pressure, and heart rate during sleep. Sleep Breath. 2017;21:497-503.

38. Hardy De Buisseret FX, Mairesse O, Newell J, Verbanck P, Neu D. While Isolated Periodic Limb Movement Disorder Significantly Impacts Sleep Depth and Efficiency, Co-Morbid Restless Leg Syndrome Mainly Exacerbates Perceived Sleep Quality. Eur Neurol. 2017;77:272-80.

39. Lavie L. Oxidative stress in obstructive sleep apnea and intermittent hypoxia--revisited--the bad ugly and good: implications to the heart and brain. Sleep Med Rev. 2015;20:27-45.

40. Drager LF, Li J, Shin MK, Reinke C, Aggarwal NR, Jun JC, et al. Intermittent hypoxia inhibits clearance of triglyceride-rich lipoproteins and inactivates adipose lipoprotein lipase in a mouse model of sleep apnoea. Eur Heart J. 2012;33(6):783-90

41. Phillips CL, Yee BJ, Marshall NS, Liu PY, Sullivan DR, Grunstein RR. Continuous positive airway pressure reduces post-prandial lipidemia in obstructive sleep apnea. A randomized, placebo-controlled crossover trial. Am J Respir Crit Care Med. 2011;184:355-61.

42. $\mathrm{Xu} \mathrm{H}, \mathrm{Yi} \mathrm{H}$, Guan J, Yin S. Effect of continuous positive airway pressure on lipid profile in patients with obstructive sleep apnea syndrome: a metaanalysis of randomized controlled trials. Atherosclerosis. 2014;234(2):446-53.

43. Tassone F, Lanfranco F, Gianotti L, Pivetti S, Navone F, Rossetto R, et al. Obstructive sleep apnoea syndrome impairs insulin sensitivity independently of anthropometric variables. Clin Endocrinol. 2003;59(3):374-9.

44. Vgontzas AN. Does obesity play a major role in the pathogenesis of sleep apnoea and its associated manifestations via inflammation, visceral adiposity, and insulin resistance? Arch Physiol Biochem. 2008;114(4):211-23.

45. Cheng C, Thompson S, Edwards C, Khan N, Holscher H. Dietary Patterns and Metabolic Syndrome Among Adults with Overweight and Obesity (FS1803-19). Curr Dev Nutr. 2019;3(Suppl 1):1.
46. Davies MJ, Baer DJ, Judd JT, Brown ED, Campbell WS, Taylor PR. Effects of moderate alcohol intake on fasting insulin and glucose concentrations and insulin sensitivity in postmenopausal women: a randomized controlled trial. JAMA. 2002:287:2559-62.

47. Tsumura K, Hayashi T, Suematsu C, Endo G, Fuji S, Okada K. Daily alcohol consumption and the risk of type 2 diabetes in Japanese men: the Osaka Health Survey. Diabetes Care. 1999;22:1432-7.

48. Nova E, San Mauro-Martín I, Díaz-Prieto LE, Marcos A. Wine and beer within a moderate alcohol intake is associated with higher levels of $\mathrm{HDL}-\mathrm{C}$ and adiponectin. Nutr Res. 2019;63:42-50.

49. Klatsky AL. Alcohol and cardiovascular diseases: where do we stand today? J Intern Med. 2015;278:238-50

50. O'Keefe JH, Bhatti SK, Bajwa A, DiNicolantonio JJ, Lavie CJ. Alcohol and cardiovascular health: the dose makes the poison or the remedy. Mayo Clin Proc. 2014:89(3):382-93.

\section{Publisher's Note}

Springer Nature remains neutral with regard to jurisdictional claims in published maps and institutional affiliations.

Ready to submit your research? Choose BMC and benefit from:

- fast, convenient online submission

- thorough peer review by experienced researchers in your field

- rapid publication on acceptance

- support for research data, including large and complex data types

- gold Open Access which fosters wider collaboration and increased citations

- maximum visibility for your research: over $100 \mathrm{M}$ website views per year

At $\mathrm{BMC}$, research is always in progress.

Learn more biomedcentral.com/submissions 